\title{
The Automorphism Group of the Fibonacci Poset: A “Not Too Difficult" Problem of Stanley from 1988
}

\author{
JONATHAN DAVID FARLEY \\ Department of Applied Mathematics, Massachusetts Institute of Technology, Cambridge, Massachusetts 02139, \\ USA \\ SUNGSOON KIM \\ kim@math.jussieu.fr; http://www.math.jussieu.fr/ kim \\ Université de Picardie Jules-Verne, F-80039 Amiens, France; Institut de Mathématiques de Jussieu-Chevaleret \\ (CNRS UMR 7586), Université Paris 7, Case 7012, 2 Place Jussieu, F-75251 Paris, Cédex 05, France
}

Received April 24, 2001; Revised April 8, 2003; Accepted April 17, 2003

Abstract. All of the automorphisms of the Fibonacci poset $Z(r)$ are determined $(r \in \mathbb{N})$. A problem of Richard P. Stanley from 1988 is thereby solved.

Keywords: Fibonacci poset, Fibonacci lattice, differential poset, automorphism group, (partially) ordered set

2000 Mathematics Subject Classification: 06C05, 06B99, 06A11, 05E25, 05E10, 08A35, 20B25, 20B27, 20B30, 06A30, 06A20

\section{Introduction-To be young again}

Young's lattice $Y$, the set of Ferrers shapes partially ordered in a certain fashion, is a poset of tremendous combinatorial significance. As is well known, it is closely connected with the representation theory of the symmetric groups $S_{r}(r \in \mathbb{N})$.

In [9], Richard P. Stanley investigated lattices that share many of the interesting combinatorial properties of $Y$, the Fibonacci posets $Z(r)(r \in \mathbb{N})$. These are posets whose elements are finite words generated from the alphabet $\left\{1_{1}, 1_{2}, \ldots, 1_{r}, 2\right\}$, where one defines the covering relation as follows: $y$ covers $x$ in $Z(r)$ if either

(1) $x=2^{k} v$ and $y=2^{k} 1_{i} v$, or

(2) $x=2^{k} 1_{i} v$ and $y=2^{k} 2 v$

for some $i \in\{1,2, \ldots, r\}$ and $v \in Z(r)$. In other words, one can either delete the first 1 or convert a 2 into a 1 (as long as no other 1's appear before it). See figures 1 and 3.

The results in this paper were first obtained by the second author, Dr. SungSoon Kim, and then independently obtained by the first author. The first author would therefore like to thank Dr. SungSoon Kim for graciously inviting him to co-author this paper. The first author was supported by (U.S.) National Science Foundation Grant DMS-9971352. 


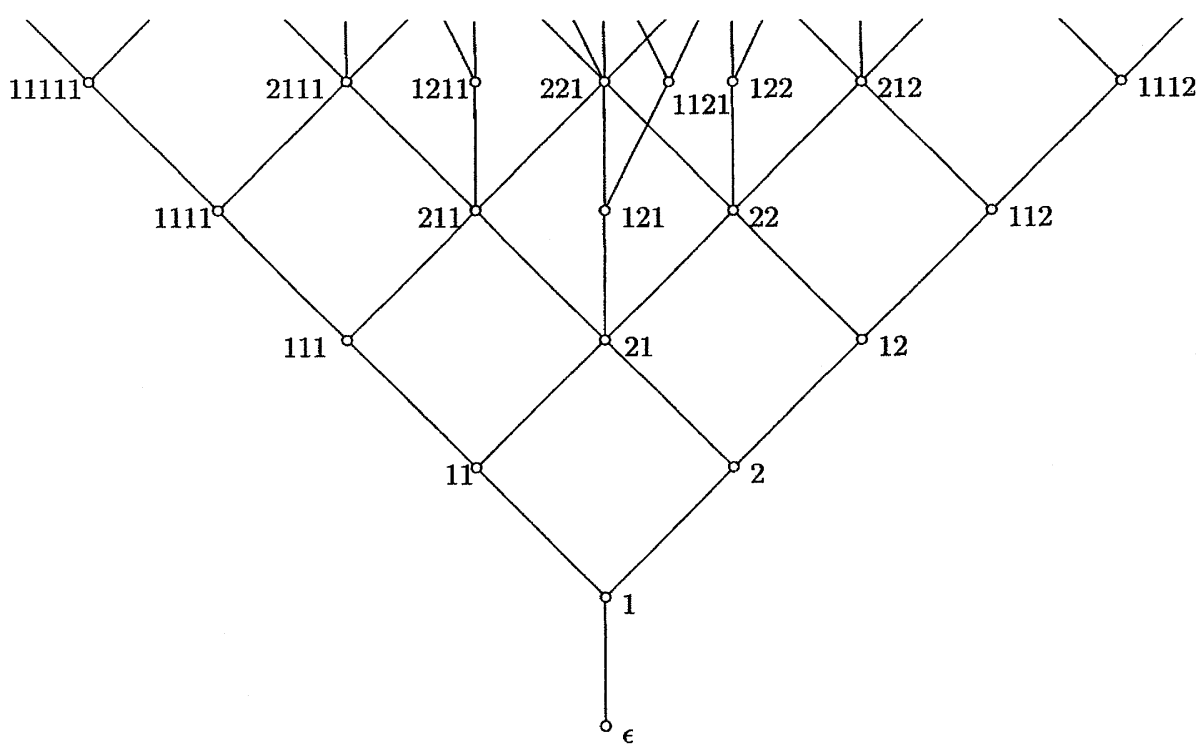

Figure 1. The Fibonacci poset $Z(1)$.

In [9], Problem 7, Stanley asks:

Problem ([9]) What is the automorphism group Aut $(Z(r))$ of the Fibonacci poset $Z(r)$ ?

(Admittedly, Stanley adds, "We suspect that Problem 7 should not be too difficult.")

We solve the problem by explicitly determining all of the automorphisms of $Z(r)$ (Theorem 4.5).

\section{Definitions}

Throughout this paper, $\mathrm{r}$ will denote a positive integer.

For basic terminology, see [2].

Let $P$ be a poset; let $p, q \in P$. We say $q$ covers $p$ (denoted $p \lessdot q$ ) if $p<q$ and for all $r \in P, p \leq r<q$ implies $p=r ; p$ is a lower cover of $q$ and $q$ is an upper cover of $p$. An element is join-irreducible if it has exactly one lower cover; the set of such elements is denoted $\mathcal{J}(P)$.

A poset with a least element is locally finite if, for all $p \in P$, there are only finitely many elements of $P$ below $p$. The rank of an element in such a poset is one less than the size of the largest chain whose top element is $p$.

In a lattice $L$, the least upper bound of $x, y \in L$ is denoted $x \vee y$.

An automorphism $\bar{\tau}$ of a poset $P$ is an order-preserving bijection whose inverse is also order-preserving. Equivalently, if $P$ is locally finite, a bijection $\bar{\tau}: P \rightarrow P$ is an automorphism if, for all $p, q \in P, p \lessdot q$ implies $\bar{\tau}(p) \lessdot \bar{\tau}(q)$ and vice versa. 
Let $\epsilon$ denote the empty word. The length $|w|$ of a word $w$ is the number of symbols in a reduced form of $w$.

If $G$ and $X$ are sets, $G^{X}$ denotes the set of tuples $\left(g_{x}\right)_{x \in X}$ where $g_{x} \in G$ for all $x \in X$. Let $S_{r}$ denote the symmetric group on $r$ letters.

\section{Facts about the Fibonacci poset $Z(r)$}

The following facts come from [9], Section 5:

The Fibonacci poset $Z(r)$ is a locally finite modular lattice with least element $\epsilon$. The rank of a word is the sum of its "letters." The lattice $Z(r)$ has the additional property that, if $w \in Z(r)$ has exactly $k$ lower covers, then it has exactly $k+r$ upper covers. These facts make $Z(r)$ an $r$-differential poset.

Indeed, $Z(r)$ is the only $r$-differential (locally finite, modular) lattice such that every complemented interval has rank at most 2 . It can be constructed inductively by "reflection" ([9], Section 6): One constructs $Z(r)$ rank by rank, reflecting the last layer one has built, then adding $r$ new join-irreducible upper covers for each element [figures 2(a)-(c)].

One deduces that, for $Z(1)$, the number of elements of each rank is a Fibonacci number.

In [9], Section 6, Stanley observes that the symmetric group $S_{r}$ acts on $Z(r)$.

Example 3.1 The 3-cycle $\sigma=(123)$ induces an automorphism $\bar{\sigma}: Z(3) \rightarrow Z(3)$ which sends, for instance, $w=1_{1} 1_{2} 21_{1} 221_{3}$ to $\bar{\sigma}(w)=1_{2} 1_{3} 21_{2} 221_{1}$. by

"However," Stanley writes, “ $Z(1)$ has (at least) an additional automorphism $\omega$, defined

$$
\begin{aligned}
\omega(v 11) & =v 2 \\
\omega(v 2) & =v 11 \\
\omega(w) & =w \quad \text { otherwise" }
\end{aligned}
$$

[i.e., if $w$ is not of the form $v 11$ or $v 2$ for some $v \in Z(1)]$. The fact that $\omega$ is an automorphism is "obvious" by reflecting figure 1 about the vertical axis; but we prove it rigorously in Lemma 4.4.

Our list of references contains other papers dealing with the Fibonacci poset.

\section{The automorphism group of the Fibonacci poset $Z(r)$ : The solution to Stanley's problem}

In this section we solve Stanley's problem (see Section 1) by finding all of the automorphisms of $Z(r)$ (Theorem 4.5).

The idea is that each word $w \in Z(r)$ has a set $C_{w}$ of $r$ join-irreducible upper covers; and an automorphism $\bar{\tau}$ of $Z(r)$ must send $C_{w}$ to $C_{\bar{\tau}(w)}$. Hence, to each $w$ we can associate an element of $S_{r}$. As $Z(r)$ is a locally finite lattice, the action of $\bar{\tau}$ on $\mathcal{J}(Z(r))$ determines $\bar{\tau}$. 


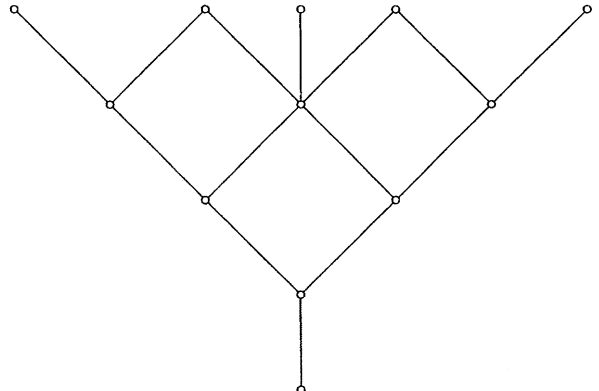

(a)

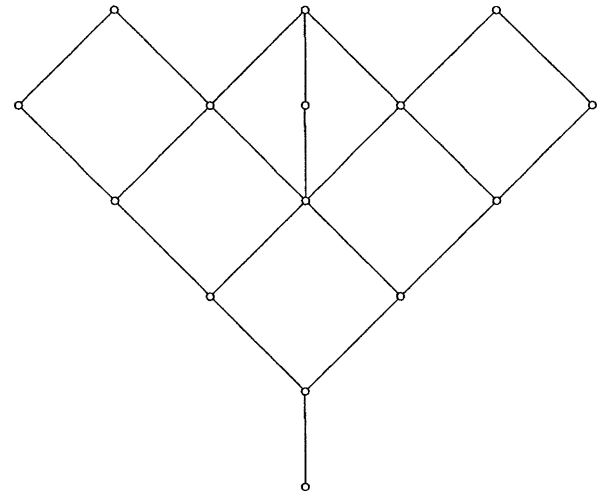

(b)

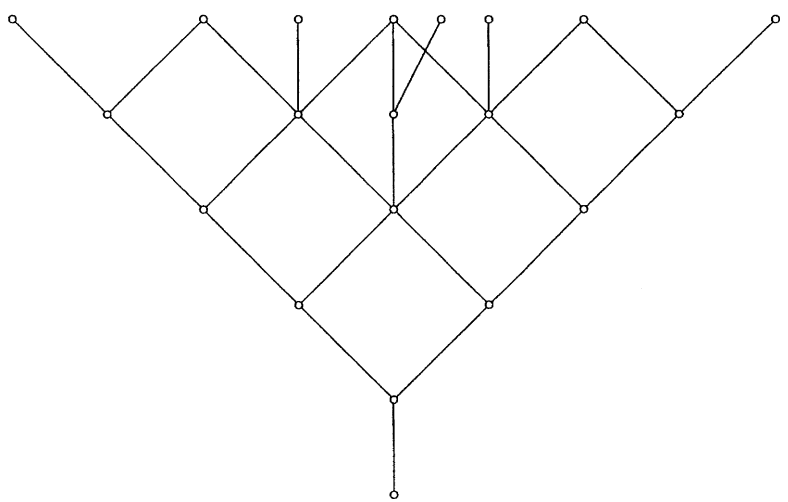

(c)

Figure 2. (a) Building $Z(r)$ by reflection. (b) Building $Z(r)$ by reflection. (c) Building $Z(r)$ by reflection.

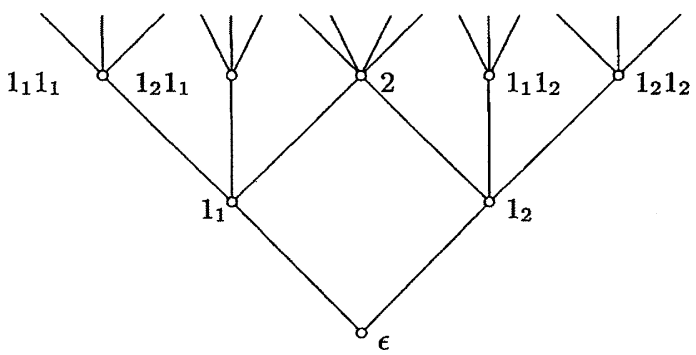

Figure 3. The Fibonacci poset $Z(2)$. 
Lemma 4.1 (Proposition 2 of [4]) The set $\mathcal{J}(Z(r))$ of join-irreducibles of $Z(r)$ is

$$
\begin{array}{r}
\left\{1_{i} v \mid 1 \leq i \leq r ; v \in Z(r)\right\} \quad \text { if } r \geq 2, \\
\{1 v \mid v \in Z(1)\} \cup\{2\} \quad \text { if } r=1 .
\end{array}
$$

Proof: Clearly each element listed is join-irreducible. Now let $w \in \mathcal{J}(Z(r))$. Suppose $w$ is not of the above form: then $w=2 v$ for some $v \in Z(r)$. If $r \geq 2$, then $w$ has at least $t w o$ lower covers, $1_{1} v$ and $1_{2} v$, a contradiction. Hence $r=1$.

If $w=21 u$ for some $u \in Z(1)$, then $w$ has the two lower covers $11 u$ and $2 u$; if $w=22 u$ for some $u \in Z(1)$, then $w$ has the two lower covers $12 u$ and $21 u$.

Lemma 4.2 Let $\vec{\tau}=\left(\tau_{w}\right)_{w \in Z(r)}$ be a fixed element of $S_{r}^{Z(r)}$. Define a map $\bar{\tau}: Z(r) \rightarrow Z(r)$ inductively on the length of the argument, as follows: For all $w \in Z(r)$, let

$$
\bar{\tau}(w)= \begin{cases}2^{k} & \text { if } w=2^{k}(k \geq 0), \\ 2^{k} 1_{\tau_{v}(i)} \bar{\tau}(v) & \text { if } w=2^{k} 1_{i} v[k \geq 0 ; 1 \leq i \leq r ; v \in Z(r)] .\end{cases}
$$

Then $\bar{\tau}$ belongs to Aut $(Z(r))$ and $\bar{\tau}(2)=2$.

Proof: It is clear that $\bar{\tau}$ is a bijection whose inverse is $\bar{v}$ for some $\vec{v} \in S_{r}^{Z(r)}$. Thus it suffices to prove that if $x, y \in Z(r)$ and $x \ll y$, then $\bar{\tau}(x) \ll \bar{\tau}(y)$.

It is obvious that, for all $v \in Z(r), \bar{\tau}(2 v)=2 \bar{\tau}(v)$.

Case 1. $x=2^{k} v$ and $y=2^{k} 1_{i} v[k \geq 0 ; 1 \leq i \leq r ; v \in Z(r)]$

Then $\bar{\tau}(x)=2^{k} \bar{\tau}(v)$ and $\bar{\tau}(y)=2^{k} 1_{\tau v(i)} \bar{\tau}(v)$, so $\bar{\tau}(x) \lessdot \bar{\tau}(y)$.

Case 2. $x=2^{k} 1_{i} v$ and $y=2^{k} 2 v[k \geq 0 ; 1 \leq i \leq r ; v \in Z(r)]$

Then $\bar{\tau}(x)=2^{k} 1_{\tau_{v}(i)} \bar{\tau}(v)$ and $\bar{\tau}(y)=2^{k} 2 \bar{\tau}(v)$, so $\bar{\tau}(x) \lessdot \bar{\tau}(y)$.

Let $\hat{\tau} \in$ Aut $(Z(r))$ be such that $\hat{\tau}(2)=2$. Since $\hat{\tau}$ maps $\mathcal{J}(Z(r))$ bijectively onto itself, but fixes 2 , then, by Lemma 4.1, for all $w \in Z(r)$ and $1 \leq i \leq r, \hat{\tau}\left(1_{i} w\right)=1_{j} v$ for some $j \in\{1,2, \ldots, r\}$ and $v \in Z(r)$. We will show that $v$ depends only on $w$ and not on $i$ :

Lemma 4.3 Let $\hat{\tau} \in$ Aut $(Z(r))$ be such that $\hat{\tau}(2)=2$. For all $w \in Z(r)$, define $\tau_{w} \in S_{r}$ as follows: For $1 \leq i \leq r, \hat{\tau}\left(1_{i} w\right)=1_{\tau_{w}(i)} \hat{\tau}(w)$.

Let $\vec{\tau}=\left(\tau_{w}\right)_{w \in Z(r)}$. Define $\bar{\tau}$ as in Lemma 4.2 .

Then $\hat{\tau}=\bar{\tau}$.

Proof: From what was said before the statement of the proposition, $v$ must be $\hat{\tau}(w)$, as $w$ is the unique lower cover of $1_{i} w$, so $\hat{\tau}(w)$ is the unique lower cover of $1_{j} v$, which is $v$. Thus $\tau_{w} \in S_{r}$ is well defined. 
Claim For all $v \in Z(r), \hat{\tau}(2 v)=2 \hat{\tau}(v)$.

Proof of Claim (by induction on $|v|$ ): The claim follows from the definition of $\hat{\tau}$ if $v=\epsilon$.

Case 1. $v=1_{i} u[1 \leq i \leq r ; u \in Z(r)]$.

Then $2 u, 1_{1} 1_{i} u \lessdot 21_{i} u=2 v$, so $2 u \vee 1_{1} 1_{i} u=2 v$. By the induction hypothesis, $\hat{\tau}(2 u)=$ $2 \hat{\tau}(u)$ and we know that $\hat{\tau}\left(1_{1} 1_{i} u\right)=1_{j} 1_{k} \hat{\tau}(u)$ [where $1 \leq j, k \leq r$ and $\left.\hat{\tau}(v)=1_{k} \hat{\tau}(u)\right]$. Note that $2 \hat{\tau}(u), 1_{j} 1_{k} \hat{\tau}(u) \lessdot 21_{k} \hat{\tau}(u)$, so $2 \hat{\tau}(u) \vee 1_{j} 1_{k} \hat{\tau}(u)=21_{k} \hat{\tau}(u)$ and hence $\hat{\tau}(2 v)=$ $21_{k} \hat{\tau}(u)=2 \hat{\tau}(v)$.

Case 2. $v=2 u[u \in Z(r)]$.

Then $21_{1} u, 1_{1} 2 u \lessdot 22 u=2 v$, so $21_{1} u \vee 1_{1} 2 u=2 v$. By Case $1, \hat{\tau}\left(21_{1} u\right)=2 \hat{\tau}\left(1_{1} u\right)=$ $21_{j} \hat{\tau}(u)$ for some $j \in\{1,2, \ldots, r\}$. Also, $\hat{\tau}\left(1_{1} 2 u\right)=1_{k} \hat{\tau}(2 u)=1_{k} 2 \hat{\tau}(u)$ for some $k \in$ $\{1,2, \ldots, r\}$.

Note that $21_{j} \hat{\tau}(u), 1_{k} 2 \hat{\tau}(u) \lessdot 22 \hat{\tau}(u)$, so $21_{j} \hat{\tau}(u) \vee 1_{k} 2 \hat{\tau}(u)=22 \hat{\tau}(u)$ and hence $\hat{\tau}(2 v)=$ $22 \hat{\tau}(u)=2 \hat{\tau}(v)$,

The lemma follows, as $\hat{\tau}$ and $\bar{\tau}$ agree on all words in $Z(r)$.

The following result is asserted without proof in [9], Problem 7.

Lemma 4.4 The map $\omega$ of Section 3 belongs to Aut $(Z(1))$.

Proof: As $\omega^{2}=\mathrm{id}_{Z(1)}$, it suffices to prove that if $x, y \in Z(1)$ and $x \lessdot y$, then $\omega(x) \lessdot \omega(y)$.

Case 1. $x=2^{k} v$ and $y=2^{k} 1 v[k \geq 0 ; v \in Z(1)]$.

If $v=u 11, u 2$, or $u 21$ for some $u \in Z(1)$, then $\omega(x) \lessdot \omega(y)$. If $v=1$, then $\omega(x)=$ $\omega\left(2^{k} 1\right)=2^{k} 1 \lessdot 2^{k} 2=\omega\left(2^{k} 11\right)=\omega(y)$.

Else, $v=\epsilon$. If $k=0$, then $\omega(x)=\omega(\epsilon)=\epsilon \lessdot 1=\omega(1)=\omega(y)$. If $k \geq 1$, then $\omega(x)=\omega\left(2^{k}\right)=2^{k-1} 11 \lessdot 2^{k-1} 21=2^{k} 1=\omega\left(2^{k} 1\right)=\omega(y)$.

Case 2. $x=2^{k} 1 v$ and $y=2^{k} 2 v[k \geq 0 ; v \in Z(1)]$.

If $v=u 11, u 2, u 21$, or $\epsilon$ for some $u \in Z(1)$, then $\omega(x) \lessdot \omega(y)$.

Else, $v=1$, so $\omega(x)=\omega\left(2^{k} 11\right)=2^{k} 2 \lessdot 2^{k} 21=\omega\left(2^{k} 21\right)=\omega(y)$.

Theorem 4.5 The automorphism group of $Z(1)$ is isomorphic to $\mathbb{Z}_{2}$, the non-trivial automorphism being $\omega: Z(1) \rightarrow Z(1)$ defined for all $w \in Z(1)$ by:

$$
\omega(w)= \begin{cases}u 2 & \text { if } w=u 11[u \in Z(1)], \\ u 11 & \text { if } w=u 2[u \in Z(1)] \\ w & \text { otherwise. }\end{cases}
$$


The automorphism group of $Z(r)$ for $r \geq 2$ is in one-to-one correspondence with $S_{r}^{Z(r)}$. Given $\bar{\tau}=\left(\tau_{w}\right)_{w \in Z(r)} \in S_{r}^{Z(r)}$, define $\bar{\tau}: Z(r) \rightarrow Z(r)$ for all $w \in Z(r)$ by induction on $|w|$ :

$$
\bar{\tau}(w)= \begin{cases}2^{k} & \text { if } w=2^{k}(k \geq 0), \\ 2^{k} 1_{\tau_{v}(i)} \bar{\tau}(v) & \text { if } w=2^{k} 1_{i} v[k \geq 0 ; 1 \leq i \leq r ; v \in Z(r)] .\end{cases}
$$

In general, Aut $(Z(r))$ is not isomorphic to the product group $S_{r}^{Z(r)}$.

Proof: If $\bar{\tau} \in$ Aut $(Z(1))$, then $\bar{\tau}(2)$ is either 2 or 11 . By Lemmas 4.2 and 4.3, only the identity automorphism fixes 2 , and $w^{2}=\mathrm{id}_{Z(1)}$, so Aut $(Z(1))=\left\{\mathrm{id}_{Z(1), \omega}\right\}$.

If $r \geq 2$, then $\bar{\tau}(2)=2$ for all $\bar{\tau} \in$ Aut $(Z(r))$, since every other element of rank 2 is, by Lemma 4.1, join-irreducible. Hence the form of $\bar{\tau}$ follows from Lemmas 4.2 and 4.3.

Note that if $\bar{\tau}$ and $\bar{v}$ are automorphisms associated with the sequences $\vec{\tau}=\left(\tau_{w}\right)_{w \in Z(r)}, \vec{v}=$ $\left(v_{w}\right)_{w \in Z(r)} \in S_{r}^{Z(r)}$, and $v \in Z(r)$ is a minimal element such that $\tau_{v} \neq v_{v}$ (say, $\tau_{v}(i)=j \neq$ $\left.k=v_{v}(i)\right)$, then $\bar{\tau}\left(1_{i} v\right)=1_{j} \bar{\tau}(v) \neq 1_{k} \bar{\tau}(v)=1_{k} \bar{v}(v)=\bar{v}\left(1_{i} v\right)$. Hence, each automorphism constructed is unique.

To prove the last statement, note that $S_{2}^{Z(2)}$ has exponent 2 . But consider the map $\bar{\tau}$ : $Z(2) \rightarrow Z(2)$ where, for all $w \in Z(2)$,

$$
\tau_{w}= \begin{cases}(12) & \text { if } w=\epsilon \text { or } w=1_{1} \\ (1)(2) & \text { otherwise. }\end{cases}
$$

(See figure 3.)

Hence $\bar{\tau}\left(1_{1} 1_{1}\right)=1_{2} 1_{2}, \bar{\tau}\left(1_{2} 1_{2}\right)=1_{2} 1_{1}$, so $\bar{\tau}^{2}\left(1_{1} 1_{1}\right) \neq 1_{1} 1_{1}$ and $\bar{\tau}^{2} \neq \mathrm{id}_{Z(2)}$. Therefore Aut $(Z(2)) \nRightarrow S_{2}^{Z(2)}$.

Collecting all the observations above, we can conclude as follows.

Corollary 4.6 The automorphism group Aut $(Z(r))$ is isomorphic to $\mathbb{Z}_{2}$ ? $S_{r}$.

Proof: Let us denote the reflection of $\omega$ of $Z(1)$ and let $t_{i}=(i, i+1)(i=1, \ldots, r-1)$ permutting the $1_{i}$ 's. Then one can see that all the automorphisms of $Z(r)$ are the products of $t_{i}$ and $s$ under the following conditions:

order relations:

$$
t_{i}^{2}=s^{2}=1 \quad(i=1, \ldots, r-1)
$$

and the braid relations:

$$
\begin{aligned}
t_{i} t_{j} & =t_{j} t_{i} \quad\left(\text { for all } i, j=0, \ldots, r-1 \text { such that }|i-j|>1, t_{0}=s\right), \\
s t_{j} & =t_{j} s \quad(j=2, \ldots, r-1), s t_{1} s t_{1}=t_{1} s t_{1} s, \\
t_{i} t_{i+1} t_{i} & =t_{i+1} t_{i} t_{i+1} \quad(i=1, \ldots, r-1) .
\end{aligned}
$$


Hence we can conclude that Aut $(Z(r)) \cong \mathbb{Z}_{2}$ 2 $S_{r}$ which is the Weyl group of type $B_{r}$

Remark According to the Shephard-Todd notation, this is $G(2,1, r)$, which is a special case of the complex reflection group of type $G(d, 1, r)=\mathbb{Z}_{d}$ 2 $S_{r}$ with $d=2$.

For further detailed verifications of the relations of $s$ and $t_{i}$ in the proof above, please consult the second author.

The problem of Stanley from 1988 is thereby solved.

\section{References}

1. F. Brenti, "Log-concavity and combinatorial properties of Fibonacci lattices," European Journal of Combinatorics 12 (1991), 459-476.

2. B.A. Davey and H.A. Priestly, Introduction to Lattices and Order, 2nd edition, Cambridge University Press, Cambridge, 2002.

3. F.M. Goodman and S.V. Kerov, "The Martin boundary of the Young-Fibonacci lattice," Journal of Algebraic Combinatorics 11 (2000), 17-48.

4. R. Kemp, "Tableaux and rank-selection in Fibonacci lattices," European Journal of Combinatorics 18 (1997), 179-193.

5. D. Kremer, "A bijection between intervals in the Fibonacci posets," Discrete Mathematics 217 (2000), 225235.

6. D. Kremer and K.M. O'Hara, "A bijection between maximal chains in Fibonacci posets," Journal of Combinatorial Theory (A) 78 (1997), 268-279.

7. S. Okada, "Algebras associated to the Young-Fibonacci lattice," Transactions of the American Mathematical Society 346 (1994), 549-568.

8. R.P. Stanley, “The Fibonacci lattice,” The Fibonacci Quartely 13 (1975), 215-232.

9. R.P. Stanley, "Differential posets," Journal of the American Mathematical Society 1 (1988), 919-961.

10. R.P. Stanley, "Further combinatorial properties of two Fibonacci lattices," European Journal of Combinatorics 11 (1990), 181-188. 